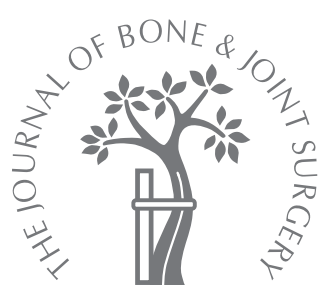
A. K. Bremer, F. Kalberer, C. W. A. Pfirrmann, C. Dora
From The University of Zürich, Zürich, Switzerland

\title{
Soft-tissue changes in hip abductor muscles and tendons after total hip replacement
}

\author{
COMPARISON BETWEEN THE DIRECT ANTERIOR AND THE \\ TRANSGLUTEAL APPROACHES
}

The direct anterior approach in total hip replacement anatomically offers the chance to minimise soft-tissue trauma because an intermuscular and internervous plane is explored. This motivated us to abandon our previously used transgluteal approach and to adopt the direct anterior approach for total hip replacement. Using MRI, we performed a retrospective comparative study of the direct anterior approach with the transgluteal approach. There were 25 patients in each group. At one year post-operatively all the patients underwent MRI of their replaced hips. A radiologist graded the changes in the soft-tissue signals in the abductor muscles. The groups were similar in terms of age, gender, body mass index, complexity of the reconstruction and absence of symptoms.

Detachment of the abductor insertion, partial tears and tendonitis of gluteus medius and minimus, the presence of peri-trochanteric bursal fluid and fatty atrophy of gluteus medius and minimus were significantly less pronounced and less frequent when the direct anterior approach was used. There was no significant difference in the findings regarding tensor fascia lata between the two approaches.

We conclude that use of the direct anterior approach results in a better soft-tissue response as assessed by MRI after total hip replacement. However, the impact on outcome needs to be evaluated further.

Persistent symptoms may remain after an otherwise successful total hip replacement (THR). According to a recent publication $26 \%$ of patients were unable to return to sports because of reasons related to joint replacement, most commonly pain. ${ }^{1}$ Possible causes include damage to muscles and tendons and their innervations. We have previously shown an association between residual pain and damage to the soft tissues in a comparative MRI study involving asymptomatic and symptomatic patients after THR. ${ }^{2}$ Defects of the abductor tendons and fatty atrophy of parts of gluteus medius and minimus were seen predominantly in symptomatic patients. Even in asymptomatic patients, some soft-tissue damage such as tendonitis, partial tendon tears and fatty atrophy of the anterior portion of gluteus minimus was found.

The term 'minimally invasive hip replacement' is open to interpretation and may reflect the different surgical goals including an attempt to minimise local soft-tissue trauma. From an anatomical perspective the anterior approach to the hip fulfils this objective by following an intermuscular and internervous plane between the superior gluteal and femoral nerves. ${ }^{3}$
In June 2004, having used the transgluteal approach $^{4}$ in THR for many years, the relationship between residual pain and soft-tissue damage as demonstrated by MRI motivated us to adopt a less invasive technique using the anterior approach..$^{5}$ Our aim in this study was to test the hypothesis that, compared with the transgluteal approach, the minimally invasive anterior approach considerably reduced damage to muscle and tendons as evaluated by MRI one year after THR.

\section{Patients and Methods}

Between February and October 2005, 145 primary THRs were performed by two surgeons (FK, CD). Because of complex pathology including high and low hip dislocations and severe post-Perthes' deformities, 11 hips were approached using a digastric trochanteric osteotomy and the remaining 134 by a minimally invasive anterior approach. During this period the transgluteal approach was not used. Of the 134 patients, 54 were excluded because they had undergone previous hip surgery or had a neurological deficit such as spinal stenosis, lumbar radiculopathy or paraplegia, all of which can alter the quality of the 
pelvitrochanteric musculature by scarring or denervation. At follow-up at one year in 2006 the remaining 80 patients were asked to undergo investigation of their hip musculature by MRI. The study had ethical approval. Only 25 patients, however, agreed to participate. Reasons for refusal included claustrophobia and the time involved in the MRI examination.

In the study group an extension-distraction table (AMIS mobile leg positioner; Medacta, Castel San Pietro, Switzerland) had been used and an uncemented prosthesis (Quadra-H stem and Versafit-CC acetabular component; Medacta) implanted. In order to avoid injury to the lateral cutaneous nerve of the thigh and to tensor fascia lata, the skin incision was centred on the top of tensor fascia lata and its fascial sheath entered before blunt dissection along its medial border was performed. A U-shaped capsulotomy based laterally was then made to protect tensor fascia lata followed by the anchoring of a soft-tissue retractor within the joint capsule. After resection of the femoral head and implantation of the acetabular component the leg was secured in about $20^{\circ}$ to $30^{\circ}$ of extension, $90^{\circ}$ of external rotation and $20^{\circ}$ of adduction using the leg positioner for preparation of the femoral canal. The broaches used were the straight Judet type ${ }^{3}$ and did not have offset handles. Wound closure comprised a continuous suture of the fascial sheath of the tensor fascia lata, the subcutaneous layer and the skin. On the day after surgery the patients began to mobilise on two crutches, bearing weight as tolerated, for two weeks. After this period walking without supports was allowed.

A control group consisted of 25 highly selected patients who had undergone primary THR using a transgluteal approach. They underwent MRI studies one year postoperatively. For inclusion the patients had to report no hip pain at rest, during physical activity or on local palpation of the greater trochanter. Additionally, they had to show the same abductor strength as their opposite non-operated hip as measured using an Isobex machine (Medical Device Solutions AG, Oberburg, Switzerland). They had to have no limp, not have undergone any previous hip surgery, and have no neuromuscular disorder. They also gave informed consent to participate and undergo MRIs. This control group had originally been selected for our previous MRI study investigating soft-tissue damage in patients after THR. ${ }^{2}$ They had undergone THR between November 2001 and May 2002 by two surgeons, one of whom (CD) was involved in the current study. A hybrid THR system (cemented Exafit stem and uncemented Fitmore acetabular component; Zimmer, Winterthur, Switzerland) had been used in this group. These operations had been performed with the patient in the lateral position. The fibres of gluteus medius and vastus lateralis were split in the middle of the greater trochanter thereby developing an anterior flap according to the method of Bauer and Russe. ${ }^{4}$

Care had been taken to reattach the anterior flap using transosseous sutures (Ethibond 3; Ethicon, Spreitenbach,
Switzerland) and to protect healing by partial weightbearing for six weeks. Details of the patients in both groups are given in Table I.

MRI. The same 1.5-T system (Symphony; Siemens Medical Solutions, Erlangen, Germany) and protocol were used for all the patients in both groups. Using a flexible wrap-around receive-only surface coil, we obtained coronal T2-weighted fast spin-echo, sagittal T1-weighted spin-echo, transverse short inversion time inversion-recovery and transverse T1-weighted spin-echo MR scans. The frequency-encoding gradient was always parallel to the prosthesis. One experienced musculoskeletal radiologist (CWAP) analysed the MR scans. The abductor tendons and muscles were divided into three parts: anterior, involving the tendon of gluteus minimus; lateral, involving the lateral part of gluteus medius; and posterior, including the posterior part of the gluteus medius. Defects in the tendon such as tears or detachments, were identified as hyperintense signals extending to both surfaces of the tendon on fluid-sensitive MR sequences. The diameter of the tendon was evaluated qualitatively as normal or altered in the sense of a partial tear, that is, either thinner or thickened. Signal alterations within the tendons were rated on the T1-weighted MR scans as normal (hypo-intense) or with tendonitis (increased signal intensity compared with the normal tendon). The presence or absence of peri-trochanteric bursal fluid was assessed on fluid-sensitive MR frequencies. On the transverse T1-weighted MR scans fatty atrophy of gluteus minimus and medius was measured at two different planes at one-third and two-thirds of the distance between the iliac crest and the tip of the greater trochanter, for the anterior, middle and posterior portions. This was graded as follows: 0 , no intramuscular fat; 1 , some fatty streaks; 2 , less fat than muscle tissue; 3 , equal amounts of fat and muscle tissue; and 4, more fat than muscle tissue. ${ }^{6}$ Fatty atrophy of the tensor fascia lata was rated on its greatest section. Interobserver agreement had been assessed in a previous study and rated as excellent. ${ }^{2}$

Statistical analysis. The chi-squared test was used for analysing categorical differences between the groups for their clinical details, tendon defects and diameter, signal alterations and bursa, and odds ratio (OR) calculated. The Mann-Whitney U test was used for the comparison of continuous variables of the grading of fatty atrophy, and an unpaired two-sided $t$-test for continuous demographic variables. All statistical analyses were done using SPSS for Windows version 11.0 (SPSS Inc., Chicago, Illinois). A p-value $\leq 0.05$ was deemed to be significant.

\section{Results}

In terms of gender, age, side and body mass index (BMI) there were no significant differences between the two groups (Table I). The MRI findings are summarised in Table II. Tears or detachments of portions of the insertions of gluteus medius and minimus were not encountered in any of the patients in the study group. However, 
Table I. Details of the patients in both groups

\begin{tabular}{llll}
\hline & Study (n= 25) & Control (n= 25) & p-value \\
\hline Male:female & $10: 15$ & $14: 11$ & $0.1^{*}$ \\
Mean (range) age in years & $70(49$ to 92$)$ & $60(32$ to 88$)$ & $0.08^{\dagger}$ \\
Left:right & $12: 13$ & $13: 12$ & $0.69^{*}$ \\
Body mass index (range) in $\mathrm{kg} / \mathrm{m}^{2}$ & $26(18$ to 35$)$ & $25(18$ to 27$)$ & $0.73^{\dagger}$ \\
\hline * chi-squared test & & & \\
$\dagger$ unpaired two-sided $t$-test & & &
\end{tabular}

\begin{tabular}{|c|c|c|c|}
\hline & Study (n= 25) & Control $(n=25)$ & p-value ${ }^{*}$ \\
\hline Bursal fluid & 2 & 8 & 0.034 \\
\hline \multicolumn{4}{|l|}{ Tendinosis } \\
\hline Anterior portion & 5 & 16 & 0.02 \\
\hline Lateral portion & 6 & 22 & 0.00 \\
\hline Posterior portion & 2 & 5 & 0.22 \\
\hline \multicolumn{4}{|l|}{ Partial tendon tear } \\
\hline Anterior portion & 8 & 21 & 0.00 \\
\hline Lateral portion & 5 & 24 & 0.00 \\
\hline Posterior portion & 0 & 1 & 0.31 \\
\hline \multicolumn{4}{|c|}{ Full-thickness tendon tear } \\
\hline Anterior portion & 0 & 2 & 0.14 \\
\hline Lateral portion & 0 & 4 & 0.03 \\
\hline Posterior portion & 0 & 0 & \\
\hline
\end{tabular}

* chi-squared test

in the control group such tears were present twice in the anterior portion and four times in the lateral portion of the abductor insertion. Alterations within the insertion of the abductor into the greater trochanter such as thinning or thickening and an abnormal signal of the tendon tissue were seen in both groups, especially within the anterior and lateral portion, but significantly less so in the study group. According to the OR the risk for such changes increased by a factor of seven to 96 when a transgluteal approach was used instead of a direct anterior approach. Similarly, collections of bursal fluid within the trochanteric region were significantly less common in the study group and the risk was five times higher after a transgluteal approach. The grade of fatty atrophy of muscle at different sites within the gluteus minimus and medius is summarised in Table III. Fatty atrophy of muscle within gluteus medius was significantly less pronounced in the study group than in the control group, especially within the anterior and lateral portion and at a distance of one-third between the greater trochanter and the iliac crest. The same was observed for gluteus minimus, especially within the onethird distance of the anterior portion and within the lateral portion. Fatty atrophy of tensor fascia lata muscle was similar in both groups (Mann-Whitney $U$ test, $\mathrm{p}=0.43$; Table III).

\section{Discussion}

These results confirmed our hypothesis that, when compared with the transgluteal approach, a direct anterior approach for THR results in few alterations in the MR appearance of the pelvitrochanteric muscles and tendons. Our study design had inherent limitations, not least of which was the lack of randomisation. Unfortunately, approval of the Institutional Review Board for a prospective study was extensively delayed. In the interval the use of the anterior approach became successful, and subsequent referrals for primary THR often included a specific request for an anterior approach. Secondly, MRI of the hip musculature was not obtained before surgery. Thus, some degenerative damage to tendons and muscles may have been present as part of the arthritic process before surgery. Thirdly, different implants were used in the study and control groups which could potentially have introduced bias. However, the femoral components used had a similar geometry, making it unlikely that the components themselves would have had an effect on the soft tissues during implantation. Additionally, the difference in the artefacts on MRI caused by the components were deemed to be neglectable by our radiologist. Fourthly, intra-observer agreement was not assessed. Given the excellent interobserver agreement in a previous study, ${ }^{2}$ assessment of intra-observer agreement was deemed unnecessary. Lastly, systematic evaluation of subjective and objective outcome parameters was not 
Table III. The mean (range) counts for fatty atrophy on MRI scans at a plane one-third and two-thirds of the distance between the iliac crest and the tip of the greater trochanter, for gluteus minimus and medius and at the greatest section of the tensor fascia lata

\begin{tabular}{|c|c|c|c|c|}
\hline & & Study $(n=25)$ & Control (n = 25) & p-value ${ }^{*}$ \\
\hline \multicolumn{5}{|l|}{ Gluteus minimus } \\
\hline \multirow[t]{3}{*}{ One-third distance } & Anterior portion & 1.32 (0 to 4$)$ & $2.24(0$ to 4$)$ & 0.001 \\
\hline & Lateral portion & 0.92 (0 to 3$)$ & $1.84(0$ to 4$)$ & 0.027 \\
\hline & Posterior portion & $0.80(0$ to 3$)$ & 0.88 (0 to 4$)$ & 0.714 \\
\hline \multirow[t]{3}{*}{ Two-thirds distance } & Anterior portion & 0.80 (0 to 4$)$ & $1.24(0$ to 4$)$ & 0.070 \\
\hline & Lateral portion & 0.88 (0 to 3$)$ & $1.76(0$ to 4$)$ & 0.039 \\
\hline & Posterior portion & $0.44(0$ to 3$)$ & $1.00(0$ to 4$)$ & 0.101 \\
\hline \multicolumn{5}{|l|}{ Gluteus medius } \\
\hline \multirow[t]{3}{*}{ One-third distance } & Anterior portion & 0.64 (0 to 3 ) & 0.84 (0 to 3 ) & 0.263 \\
\hline & Lateral portion & $0.20(0$ to 1$)$ & 0.52 (0 to 2 ) & 0.034 \\
\hline & Posterior portion & $0.12(0$ to 1$)$ & $0.32(0$ to 1$)$ & 0.091 \\
\hline \multirow[t]{3}{*}{ Two-thirds distance } & Anterior portion & 0.32 (0 to 3 ) & 1.04 (0 to 3 ) & 0.002 \\
\hline & Lateral portion & $0.20(0$ to 1$)$ & 1.24 (0 to 4$)$ & $<0.001$ \\
\hline & Posterior portion & $0.12(0$ to 1$)$ & $0.32(0$ to 1$)$ & 0.091 \\
\hline \multicolumn{5}{|l|}{ Tensor fascia lata } \\
\hline Greatest section & & 0.92 (0 to 2 ) & $1.10(0$ to 3$)$ & 0.430 \\
\hline
\end{tabular}

* Mann-Whitney U test

performed so that potential disadvantages of the direct anterior approach such as damage to the lateral cutaneous nerve of the thigh ${ }^{7}$ were not considered. In order to reduce bias associated with a non-randomised historical control group and the unknown pre-operative status, only highly satisfied patients were selected for the control group, which otherwise did not differ from the study group in terms of age, gender, BMI and the complexity of the procedure. Additionally, patients who had previous surgery or trauma to the hip or who had neuromuscular disease were excluded. Therefore, we believe that despite these methodological limitations, our study confirmed our hypothesis that less soft-tissue damage is produced when a direct anterior approach is used.

Although an association between soft-tissue alterations on MRI and residual symptoms after THR has been suggested, ${ }^{2,8}$ review of the current literature on minimally invasive approaches for THR reveals that there is only a short-term advantage in outcome compared with the use of other approaches. ${ }^{9}$ Our study only investigated changes detected by MRI and the impact of the soft-tissue alterations on outcome needs further investigation.
No benefits in any form have been received or will be received from a commercial party related directly or indirectly to the subject of this article.

\section{References}

1. Wylde V, Blom A, Dieppe P, Hewlett S, Learmonth I. Return to sport after joint replacement. J Bone Joint Surg [Br] 2008;90-B:920-3.

2. Pfirrmann CW, Nötzli HP, Dora C, Hodler J, Zanetti M. Abductor tendons and muscles assessed at MR imaging after total hip arthroplasty in asymptomatic and symptomatic patients. Radiology 2005;235:969-76.

3. Judet $\mathbf{J}$, Judet H. Anterior approach in total hip arthroplasty. Presse Med 1985; 14:1031-3 (in French).

4. Bauer R, Russe W. The transgluteal approach in hip joint arthroplasty. Z Orthop Ihre Grenzgeb 1984;122:48-9 (in German).

5. Matta JM, Shahrdar C, Ferguson T. Single-incision anterior approach for total hip arthroplasty on an orthopaedic table. Clin Orthop 2005;441:115-24.

6. Zanetti M, Gerber C, Hodler J. Quantitative assessment of the muscles of the rotator cuff with magnetic resonance imaging. Invest Radiol 1998;33:163-70.

7. Bhargava T, Goytia RN, Jones LC, Hungerford MW. Lateral cutaneous nerve impairment after direct anterior approach for total hip arthroplasty. Orthopedics 2010;33:472-6.

8. Miozzari HH, Dora C, Clark JM, Nötzli HP. Late repair of abductor avulsion after the transgluteal approach for hip arthroplasty. J Arthroplasty 2010;25:450-7.

9. Wall SJ, Mears SC. Analysis of published evidence on minimally invasive total hip arthroplasty. J Arthroplasty 2008;23:55-8. 